\title{
EXPERIMENTAL STUDIES ON HEMOLYTIC DISEASE
}

\author{
YUKIO NAGAKI \\ Department of Legal Medicine, School of Medicine, \\ Keio University
}

(Received on May 24, 1952)

I. Studies on the influences of the anti-rat-erythrocyte, -serum, -bone marrow and -liver rabbit serum to newborn rats and adult rats.

\section{INTRODUCTION}

In 1940, Landsteiner and Wiener found the Rh-factor of human red cells. Levin and his associates, in 1941, found that the $\mathrm{Rh}$-factor has a relation to erythroblastosis. Since then, studies on hemolytic disease have become active. D. W. Bruner et al. ${ }^{(1)}$ reported on the hemolytic disease produced by isoimmunization in baby pigs. L. E. Young et al. ${ }^{(2,3)}$ and R. M. Christian et al. ${ }^{(4)}$ reported on the hemolytic disease produced by isoimmunization in newborn dogs. Bessis and Frexia ${ }^{(5)}$ produced hemolytic disease in newborn rats by giving the rabbit-anti-rat serum perorally. In order to clarify the entity of the human hemolytic disease, the author has studied grossly and histologically the influences of the hetero-immune sera containing hemolysin between rat and rabbit to newborn rats and adult rats, particularly considering jaundice and increase of erythroblasts in the circulating blood and bone marrow.

\section{EXPERIMENTAL MATERIALS AND METHODS}

1. Methods of preparation anti-sera.

(a) Anti-rat-erythrocyte rabbit serum (hereafter, abridged as anti-erythrocyte serum).

The red cells from adult rats were thoroughly washed and then a $10 \%$ saline rat-erythrocyte suspension was made. This suspension was injected into the ear vein of rabbit. The anti-serum that was used in these experiments had a homelysin titer of $1: 2,000$ and an agglutinin titer of $1: 15,000$.

(b) Anti-rat-serum rabbit serum (hereafter, abridged as anti-serum serum). The serum obtained from adult rats was injected into the ear vein of a rabbit. The anti-serum that was used in these experiments had a hemolysin titer of $1: 200$ and a precipitin titer of $1: 384$ by the anti-serum dilution 
method.

(c) Anti-rat bone marrow rabbit serum (hereafter, abridged as anti-bone marrow serum).

The bone mairow obtained from adult rats was thoroughly washed and then a $10 \%$ saline bone marrow emulsion was made with a homogenizer. This emulsion was injected into the ear vein of a rabbit. The anti-serum that was used in these experiments had a hemolysin titer of $1: 80$ and a precipitin of $1: 32$ by the anti-serum dilution method.

(d) Anti-rat-liver rabbit serum (hereafter, abridged as anti-liver serum):

An adult rat was bled to death, its liver cut to pieces, washed well removing the blood elements and then a $10 \%$ saline liver emulsion was made with a homogenizer. This emulsion was injected into the ear vein of a rabbit. The anti-serum that was used in these experiments had a hemolysin titer of $1: 10$ and a precipitin titer of $1: 32$ by the anti-serum dilution method.

2. Experimental animals.

(a) The newborn rats that were used in these experiments were 4-7 days old,

- .. while those used for venous injection were 10 days old.

(b) The adult rats weighed about $200 \mathrm{~g}$ respectively.

3. Blood smears and bone marrow smears were fixed in methylalcohol and the Giemsa staining method was employed. Section specimens were fixed in Maximows' solution, while one part was fixed in 10 per cent formalin solution and then embedded in paraffin.

The staining technique included routine hematoxylineosin, Giemsa's staining and Sudan III staining.

4. On erythroblasts.

According to the author's own investigation, the numbers of erythroblasts in the circulating blood of normal newborn rats 5 days old were 1.6 to 6.0 per 100 leucocytes and those in the bone marrow were 40 to 61 per 100 leucocytes; the number of erythroblasts in the circulating blood of adlult rats were 0 per 100 leucocytes and those in the bone marrow were 3 to 18 .

\section{EXPERIMENTAL RESULTS}

11. Experiments on newborm rats.

(a) Gross and microscopic findings concerning the anti-erythrocyte serum ex- 
Table 1

Gross observations of newborn rats that were given anti-rat-erythrocyte rabbit serum and the ratio of erythroblasts to leucocytes in their circulating blood and bone marrow smears

\begin{tabular}{|c|c|c|c|c|c|c|c|c|}
\hline $\begin{array}{l}\text { Method } \\
\text { of anti- } \\
\text { serum- } \\
\text { giving } \\
\end{array}$ & Weight & $\begin{array}{c}\text { Quantity } \\
\text { of } \\
\text { anti-serum }\end{array}$ & Jaundice & Anemia & $\begin{array}{l}\text { Subcu- } \\
\text { taneous } \\
\text { bleeding }\end{array}$ & $\begin{array}{c}\text { Hemoglo- } \\
\text { binuria }\end{array}$ & \multicolumn{2}{|c|}{$\begin{array}{l}\text { Ratio of erythroblasts to } \\
\text { leucocytes in blood and } \\
\text { bone marrow smears* }\end{array}$} \\
\hline \multirow{5}{*}{ 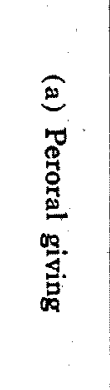 } & $8.0 \mathrm{~g}$ & $0.06 \mathrm{cc} \times 2$ & H & + & - & - & $\mathrm{H} \frac{76}{100}$ & $M \frac{97}{100}$ \\
\hline & $7.5 \mathrm{~g}$ & $0.06 \mathrm{cc} \times 2$ & H & + & - & - & $\mathrm{H} \frac{134}{100}$ & $M \frac{137}{100}$ \\
\hline & $7.0 \mathrm{~g}$ & $0.06 \mathrm{cc} \times 2$ & H & + & - & + & $\mathrm{H} \frac{127}{100}$ & $M \frac{79}{100}$ \\
\hline & $12.3 \mathrm{~g}$ & $\begin{array}{l}0.04 \mathrm{cc} \times 1 \\
0.03 \mathrm{cc} \times 2\end{array}$ & H & $\#$ & - & - & $\mathrm{H} \frac{26}{100}$. & $M \frac{100}{100}$ \\
\hline & $12.5 \mathrm{~g}$ & $\begin{array}{l}0.04 \operatorname{cc} \times 1 \\
0.03 \operatorname{cc} \times 2\end{array}$ & + & + & - & - & $H \frac{9}{100}$ & $M \frac{84}{100}$ \\
\hline \multirow{5}{*}{ 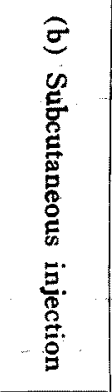 } & $8.4 \mathrm{~g}$ & $0.02 \mathrm{cc} \times 2$ & $H$ & + & H & - & $\mathrm{H} \frac{80}{100}$ & $M \frac{112}{100}$ \\
\hline & $8.5 \mathrm{~g}$ & $0.02 \mathrm{cc} \times 2$ & H & $f$ & - & + & $H \frac{62}{120}$ & $M \frac{102}{100}$ \\
\hline & $10.4 \mathrm{~g}$ & $0.01 \mathrm{cc} \times 3$ & $\#$ & $H$ & - & - & $\mathrm{H}-\frac{32}{100}$ & $M \frac{102}{100}$ \\
\hline & $10.3 \mathrm{~g}$ & $0.01 \mathrm{cc} \times 3$ & H & H & - & - & $H \frac{33}{100}$ & $M \frac{119}{100}$ \\
\hline & $10.9 \mathrm{~g}$ & $0.01 \mathrm{cc} \times 3$ & $H$ & $H$ & - & - & $\mathrm{H} \frac{25}{100}$ & $M \frac{106}{100}$ \\
\hline \multirow{3}{*}{ 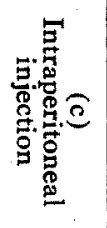 } & $10.9 \mathrm{~g}$ & $0.01 \mathrm{cc} \times 3$ & $H$ & $H$ & - & - & $\mathrm{H} \frac{103}{100}$ & $M \frac{124}{100}$ \\
\hline & $9.9 \mathrm{~g}$ & $0.01 \mathrm{cc} \times 3$ & $H$ & $H$ & - & - & $\mathrm{H} \frac{14}{100}$ & $M \frac{110}{100}$ \\
\hline & $11.3 \mathrm{~g}$ & $0.01 \mathrm{cc} \times 3$ & + & $H$ & - & - & $H-\frac{8}{100}$ & $M \frac{95}{100}$ \\
\hline \multirow{2}{*}{ 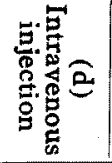 } & $12.0 \mathrm{~g}$ & $\begin{array}{l}0.01 \mathrm{cc} \times 2 \\
0.025 \mathrm{cc} \times 1\end{array}$ & + & + & - & - & $\mathrm{H} \frac{16}{100}$ & $M \frac{98}{180}$ \\
\hline & $13.3 \mathrm{~g}$ & $\begin{array}{l}0.01 \mathrm{cc} \times 2 \\
0.025 \mathrm{cc} \times 1\end{array}$ & Ht & $H$ & - & - & $H \frac{11}{100}$ & $M \frac{144}{100}$ \\
\hline
\end{tabular}

* The $\mathbf{H}$ shows the ratio of erythroblasts to leucocytes on circulating blood smears and the $M$ shows that in bone marrow smears. The denominator shows the number of leucocytes and the numerator shows that of erythroblasts.

periments are summarized in table 1 and 2. In these experiments, it was found during autopsy that all spleens but one were slightly or greatly enlarged.

(b) Gross and microscopic findings concerning the anti-bone marrow serum 
Table 2

Histologic findings in newborn rats given anti-rat-erythrocyte rabbit serum perorallay and parenterally

\begin{tabular}{|c|c|c|c|c|c|c|c|c|}
\hline \multirow{2}{*}{$\begin{array}{l}\text { Method } \\
\text { of anti- } \\
\text { serum- } \\
\text { giving }\end{array}$} & \multicolumn{3}{|c|}{ Liver } & \multicolumn{2}{|c|}{ Kidney } & \multicolumn{2}{|c|}{ Spleen } & \multirow{2}{*}{\begin{tabular}{|c|} 
Bone \\
marrow \\
Increase of \\
erythro- \\
blasts
\end{tabular}} \\
\hline & $\underset{\substack{\text { Fatty } \\
\text { tion }}}{\text { degena- }}$ & $\begin{array}{l}\text { Cloudy } \\
\text { swelling }\end{array}$ & $\begin{array}{c}\text { Increase } \\
\text { of } \\
\text { hemato- } \\
\text { poiesis }\end{array}$ & $\begin{array}{l}\text { Stagna- } \\
\text { tion of } \\
\text { glomeru- } \\
\text { lar tufts }\end{array}$ & $\begin{array}{c}\text { Cloudy } \\
\text { swelling of } \\
\text { convoluted } \\
\text { tubular- } \\
\text { epithelia } \\
\end{array}$ & $\begin{array}{c}\text { Stagna- } \\
\text { tion }\end{array}$ & $\begin{array}{c}\text { Increase } \\
\text { of } \\
\text { erythro- } \\
\text { blasts }\end{array}$ & \\
\hline \multirow{5}{*}{ 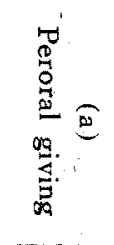 } & $+\quad \therefore$ & - & + & + & + & + & $H$ & $H$ \\
\hline & $H$ & - & - & + & H & - & - & $H$ \\
\hline & $H$ & - & + & - & $H$ & - & $H$ & $H$ \\
\hline & $H$ & - & + & - & \# & - & H & + \\
\hline & + & - & + & + & + & $H$ & H & $H$ \\
\hline \multirow{5}{*}{ 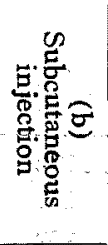 } & + & - & - & - & $H$ & $H$ & $H$ & H \\
\hline & + & + & + & - & + & - & + & H \\
\hline & \# & - & - & + & $H$ & + & $\mathrm{HH}$ & H \\
\hline & H & - & - & + & $H$ & - & $H$ & H. \\
\hline & H & - & - & + & H & - & $H$ & $H$ \\
\hline \multirow{3}{*}{ 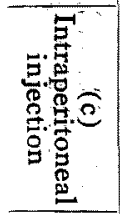 } & H & - & $H$ & + & + & - & $H$ & H \\
\hline & $H$ & - & - & + & + & + & $H$ & H. \\
\hline & H & H & - & - & $\#$ & + & $H$ & $H$ \\
\hline \multirow{2}{*}{ 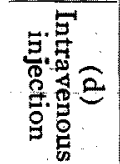 } & H & - & - & + & H & + & + & $H$ \\
\hline & H & H & - & - & H & $H$ & $H$ & $H$ \\
\hline
\end{tabular}

experiments are summarized in table 3 and 4 . In these experiments, all spleens but 3 were enlarged slightly or moderately.

(c) Gross and microscopic finding concerning the anti-serum serum experiments are summarized in table 5 and 6 . In these experiments, the majority of the spleens were enlarged slightly or moderately.

(d) Gross and microscopic findings concerning the anti-liver serum experiments are summarized in table 7 and 8 . In these experiments, no enlargement of spleens was found.

2. Experiments on adult rats.

(a) Gross and microscopic findings concerning the experiments of peroral giving of anti-erythrocyte serum are summarized in table 9 (a) and 10 (a). In these experiments all spleens were greatly enlarged, 
Table 3

Gross observations of newborn rats that were given anti-rat-bone marrow rabbit serum and the ratio of erythroblasts to leucocytes in their circulating blood and bone marrow smears

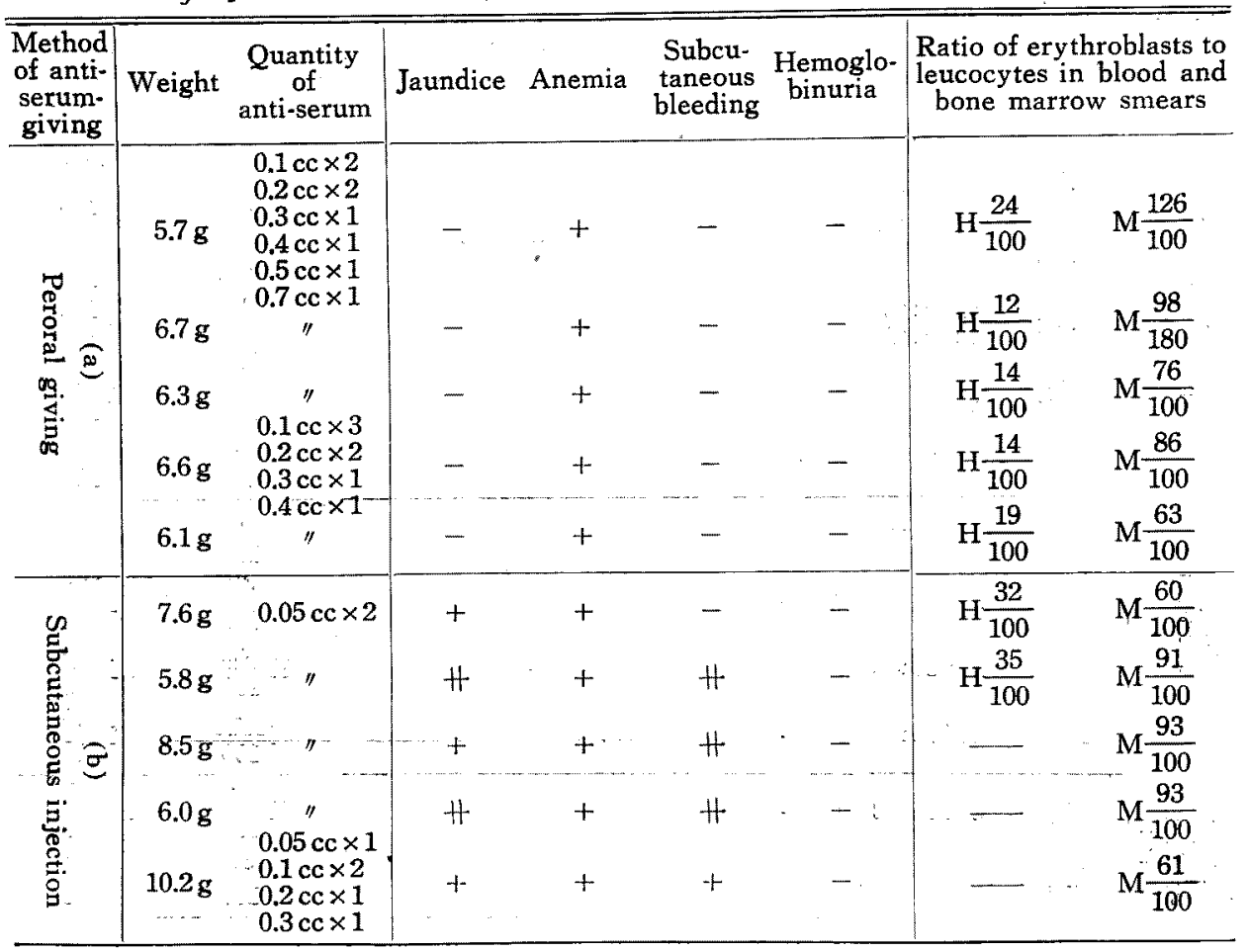

Table 4

Histologic findings in newborn rats given anti-rat-bone marrow rabbit serum perorally and subcutaneously

\begin{tabular}{|c|c|c|c|c|c|c|c|c|}
\hline \multirow[b]{2}{*}{$\begin{array}{l}\text { Method } \\
\text { of anti- } \\
\text { serum- } \\
\text { giving }\end{array}$} & \multicolumn{3}{|c|}{ Liver } & \multicolumn{2}{|c|}{ Kidney } & \multicolumn{2}{|c|}{ Spleen } & \multirow{2}{*}{$\begin{array}{c}\text { Bone } \\
\text { marrow } \\
\begin{array}{c}\text { Inerease of } \\
\text { erythro- } \\
\text { blasts }\end{array}\end{array}$} \\
\hline & $\underset{\substack{\text { Fatty } \\
\text { tionen }}}{\text {. }}$ & $\begin{array}{l}\text { Cloudy } \\
\text { swellig }\end{array}$ & $\begin{array}{c}\text { Inerease } \\
\text { of } \\
\text { hemato. } \\
\text { poiesis }\end{array}$ & $\begin{array}{l}\text { Stagna- } \\
\text { tion of } \\
\text { glomeru- } \\
\text { lar tufts }\end{array}$ & $\begin{array}{c}\text { Cloudy } \\
\text { swelling of } \\
\text { convoluted } \\
\text { tubular } \\
\text { epithelia }\end{array}$ & $\begin{array}{c}\text { Stagna- } \\
\text { tion }\end{array}$ & $\begin{array}{c}\text { Inerease } \\
\text { of } \\
\text { erythro- } \\
\text { blasts }\end{array}$ & \\
\hline \multirow{5}{*}{ 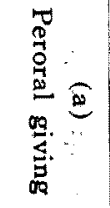 } & $H:$ & - & - & - & - & - & - & $H$ \\
\hline & + & - & - & + & $H$ & + & + & $H$ \\
\hline & $H$ & + & - & - & $H$ & + & $H$ & H \\
\hline & $H$ & - & - & + & + & + & + & H \\
\hline & $H$ & - & - & - & + & + & + & + \\
\hline \multirow{5}{*}{ 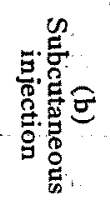 } & $H$ & - & - & + & $H$ & + & + & - \\
\hline & & - & H & + & $H$ & H & $H$ & H \\
\hline & + & $H$ & - & - & $H$ & - & + & H \\
\hline & + & $=$ & - & + & + & 卅 & + & $H$ \\
\hline & + & - & - & - & + & $H$ & \# & + \\
\hline
\end{tabular}


Table 5

Gross observations of newborn rats that were given anti-rat-serum rabbit serum and ratio of erythroblasts to leucocytes in their circulating blood and bone marrowe smears

\begin{tabular}{|c|c|c|c|c|c|c|c|c|}
\hline $\begin{array}{l}\text { Method } \\
\text { of anti- } \\
\text { serum- } \\
\text { giving }\end{array}$ & Weight & $\begin{array}{l}\text { Quantity } \\
\text { of } \\
\text { anti-serum }\end{array}$ & Jaundice & Anemia & $\begin{array}{l}\text { Subcu- } \\
\text { taneous } \\
\text { bleeding }\end{array}$ & $\begin{array}{c}\text { Hemoglo- } \\
\text { binuria }\end{array}$ & $\begin{array}{l}\text { Ratio of } \\
\text { leucocy te } \\
\text { bone ma }\end{array}$ & $\begin{array}{l}\text { aboblasts to } \\
\text { blood and } \\
\text { w smears }\end{array}$ \\
\hline & $10.1 \mathrm{~g}$ & $\begin{array}{l}0.1 \mathrm{cc} \times 2 \\
0.2 \mathrm{cc} \times 2 \\
0.4 \mathrm{cc} \times 1 \\
0.75 \mathrm{cc} \times 1\end{array}$ & - & + & - & - & $\mathrm{H} \frac{15}{100}$ & $\mathrm{M} \frac{53}{100}$ \\
\hline & $10.3 \mathrm{~g}$ & " & - & + & - & - & $H \frac{7}{100}$ & $M \frac{43}{100}$ \\
\hline$\stackrel{\theta}{\theta}$ & $8.6 \mathrm{~g}$ & $\begin{array}{l}0.1 \mathrm{cc} \times 2 \\
0.2 \mathrm{cc} \times 1\end{array}$ & + & + & - & - & $\mathrm{H} \frac{14}{100}$ & $M \frac{106}{100}$ \\
\hline$\underset{0}{5}$ & $8.1 \mathrm{~g}$ & $\begin{array}{l}0.3 \mathrm{cc} \times 1 \\
0.4 \mathrm{cc} \times 1\end{array}$ & - & + & - & - & $\mathrm{H} \frac{2}{100}$ & $M \frac{108}{100}$ \\
\hline & $7.6 \mathrm{~g}$ & $"$ & - & + & - & - & $\mathrm{H} \frac{11}{100}$ & $\mathrm{M} \frac{90}{100}$ \\
\hline & $8.6 \mathrm{~g}$ & $0.05 \mathrm{cc} \times 3$ & + & + & + & - & $\mathrm{H} \frac{7}{100}$ & $M \frac{92}{100}$ \\
\hline 点·号 & $8.1 \mathrm{~g}$ & & H & + & - & - & $\mathrm{H} \frac{45}{100}$ & $M \frac{105}{100}$ \\
\hline 疍里 & $9.0 \mathrm{~g}$ & $0.05 \mathrm{cc} \times 2$ & $H$ & + & - & - & $H \frac{5}{100}$ & $M \frac{100}{100}$ \\
\hline & $8.6 \mathrm{~g}$ & $0.05 \mathrm{cc} \times 3$ & H & + & - & - & $H \frac{9}{100}$ & $\mathrm{M} \frac{100}{100}$ \\
\hline 点. & $4.7 \mathrm{~g}$ & $0.04 \mathrm{cc} \times 2$ & $H$ & + & - & - & $\mathrm{H} \frac{34}{100}$ & $M \frac{95}{100}$ \\
\hline 要. & $8.2 \mathrm{~g}$ & $"$ & $H$ & + & - & + & $H \frac{31}{100}$ & $M \frac{106}{100}$ \\
\hline
\end{tabular}

Table 6 Histologic findings in newborn rats given anti-rat-serum rabbit serum perorally and parenterally

\begin{tabular}{|c|c|c|c|c|c|c|c|c|}
\hline \multirow[b]{2}{*}{$\begin{array}{l}\text { Method } \\
\text { of anti- } \\
\text { serum- } \\
\text { giving }\end{array}$} & \multicolumn{3}{|c|}{ Liver } & \multicolumn{2}{|c|}{ Kidney } & \multicolumn{2}{|c|}{ Spleen } & \multirow{2}{*}{$\begin{array}{c}\text { Bone } \\
\text { marrow } \\
\text { Inerease of } \\
\text { erythro- } \\
\text { blastosis }\end{array}$} \\
\hline & $\underset{\substack{\text { Fatty } \\
\text { degenera. }}}{\text { tion }}$ & $\begin{array}{l}\text { Cloudy } \\
\text { swelling }\end{array}$ & $\begin{array}{c}\text { Increase } \\
\text { of } \\
\text { hemato- } \\
\text { poiesis }\end{array}$ & $\begin{array}{l}\text { Stagna- } \\
\text { tion of } \\
\text { glomeru- } \\
\text { lar tufts }\end{array}$ & $\begin{array}{c}\text { Cloudy } \\
\text { swelling of } \\
\text { convoluted } \\
\text { tubular } \\
\text { epithelia }\end{array}$ & $\begin{array}{c}\text { Stagna- } \\
\text { tion }\end{array}$ & $\begin{array}{c}\text { Inerease } \\
\text { of } \\
\text { ery thro- } \\
\text { blastosis }\end{array}$ & \\
\hline \multirow{5}{*}{ 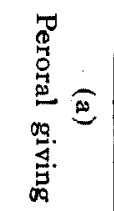 } & $H$ & - & - & $H$ & $H$ & $H$ & $H$ & + \\
\hline & $H$ & - & - & + & $H$ & - & $H$ & - \\
\hline & + & - & - & $H$ & $H$ & - & $\longrightarrow$ & $H$ \\
\hline & $H$ & - & - & - & $H$ & - & - & $H$ \\
\hline & + & - & - & $H$ & $H$ & - & $\ldots$ & $H$ \\
\hline \multirow{4}{*}{ 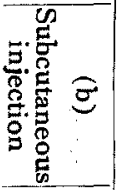 } & H & - & - & + & $H$ & $H$ & + & + \\
\hline & $H$ & - & - & - & + & - & + & $H$ \\
\hline & $H$ & $H$ & - & - & $H$ & $H$ & $H$ & $H$ \\
\hline & t & - & - & - & + & - & - & $H$ \\
\hline \multirow{2}{*}{ 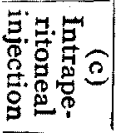 } & + & + & - & $H$ & $H$ & $\longrightarrow$ & $\longrightarrow$ & $H$ \\
\hline & $H$ & - & - & + & + & $H$ & + & $H$ \\
\hline
\end{tabular}


Table 7

Gross observations of newborn rats that were given anti-rat-liver rabbit serum and the ratio of erythroblasts to leucocytes in their circulating blood and bone marrow smears

\begin{tabular}{|c|c|c|c|c|c|c|c|c|}
\hline $\begin{array}{l}\text { Method } \\
\text { of anti- } \\
\text { serum- } \\
\text { giving }\end{array}$ & Weight & $\begin{array}{c}\text { Quantity } \\
\text { of } \\
\text { anti-serum }\end{array}$ & Jaundice & Anemia & $\begin{array}{l}\text { Subcu- } \\
\text { taneous } \\
\text { bleeding }\end{array}$ & $\underset{\text { binuria }}{\text { Hemoglo- }}$ & \multicolumn{2}{|c|}{$\begin{array}{l}\text { Ratio of erythroblasts to } \\
\text { leucocytes in blood and } \\
\text { bone marrow smears }\end{array}$} \\
\hline \multirow{4}{*}{$\underset{0}{0}$} & $7.0 \mathrm{~g}$ & $0.3 \mathrm{cc} \times 7$ & - & - & - & 一 & $\mathrm{H} \frac{2}{100}$ & $M \frac{42}{100}$ \\
\hline & $7.2 \mathrm{~g}$ & $"$ & - & - & - & - & $\mathrm{H} \frac{3}{100}$ & $M \frac{26}{100}$ \\
\hline & $8.5 \mathrm{~g}$ & $"$ & - & - & - & - & $H \frac{6}{100}$ & $\mathrm{M} \frac{52}{100}$ \\
\hline & $10.0 \mathrm{~g}$ & $"$ & - & - & - & - & $H \frac{4}{100}$ & $M \frac{65}{100}$ \\
\hline \multirow{2}{*}{ 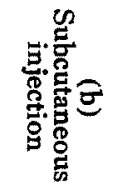 } & $7.3 \mathrm{~g}$ & $0.2 \mathrm{cc} \times 7$ & - & - & - & - & $\mathrm{H} \frac{1}{100}$ & $M \frac{27}{100}$ \\
\hline & $7.7 \mathrm{~g}$ & $"$ & - & - & - & - & $\mathrm{H} \frac{2}{100}$ & $M \frac{81}{100}$ \\
\hline
\end{tabular}

Table 8

Histolgic findings in newborn rats given anti-rat-liver rabbit serum perorally and subcutaneously

\begin{tabular}{|c|c|c|c|c|c|c|c|c|}
\hline \multirow[b]{2}{*}{$\begin{array}{l}\text { Method } \\
\text { of anti- } \\
\text { serum- } \\
\text { giving }\end{array}$} & \multicolumn{3}{|c|}{ Liver } & \multicolumn{2}{|c|}{ Kidney } & \multicolumn{2}{|c|}{ Spleen } & \multirow{2}{*}{$\begin{array}{c}\begin{array}{c}\text { Bone } \\
\text { marrow }\end{array} \\
\begin{array}{c}\text { Increase } \\
\text { of erythro- } \\
\text { blasts }\end{array}\end{array}$} \\
\hline & $\begin{array}{c}\text { Fatty } \\
\text { degenera- } \\
\text { tion }\end{array}$ & $\begin{array}{l}\text { Cloudy } \\
\text { swelling }\end{array}$ & $\begin{array}{c}\text { Increase } \\
\text { of } \\
\text { hemato- } \\
\text { poiesis }\end{array}$ & $\begin{array}{l}\text { Stagna- } \\
\text { tion of } \\
\text { glomeru- } \\
\text { lar tufts }\end{array}$ & $\begin{array}{c}\text { Cloudy } \\
\text { swelling of } \\
\text { convoluted } \\
\text { tubular } \\
\text { epithelia }\end{array}$ & $\begin{array}{c}\text { Stagna- } \\
\text { tion }\end{array}$ & $\begin{array}{l}\text { Increase } \\
\text { of } \\
\text { erythro- } \\
\text { blasts }\end{array}$ & \\
\hline \multirow{4}{*}{ 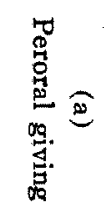 } & + & - & - & - & + & + & - & - \\
\hline & - & - & - & - & + & + & - & - \\
\hline & H & - & - & - & $H$ & - & $H$ & - \\
\hline & $H$ & - & - & - & $H$ & - & - & + \\
\hline \multirow{2}{*}{ 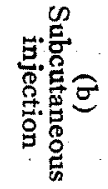 } & H & - & - & - & + & + & - & - \\
\hline & $H$ & - & - & - & \# & - & - & + \\
\hline
\end{tabular}


(b) Gross and microscopic finding concerning the experiments of subcutaneous injection of anti-erythrocyte serum are summarized in table 9 (b) and 10 (b). In these experiments, it was found that all spleens were moderately or greatly enlarged and the largest one weighed more than twice the normal.

II. Studies on the influences of the anti-rat-erythrocyte serum to newborn rats and adult rats, especially on the inhibition of jaundice.

\section{INTRODUCTION}

In the first paper, the author succeeded in causing pathologic changes very similar to the human hemolytic disease and human' "hemolytic jaundice" in newborn and adult rats by the anti-rat-erythrocyte serum. In these experiments, the author attempted to inhibit the influences of the anti-rat-erythrocyte serum to newborn and adult rats and to clarify the mechanism of the action of anti-raterythrocyte serum and hemolytic jaundice.

\section{EXPERIMENTAL MATERIALS AND METHODS}

1. In these experiments, the same anti-rat-erythrocyte serum as in the first paper was used.

2. The conditions of the newborn and adult rats were the same as in the first paper.

3. The inhibitors were injected subcutaneously 30 minutes before, 2 and 4 hours after anti-serum injection to newborn rats and 10 minutes before, 2 and 4 hours after to adult rats.

\section{EXPERIMENTAL RESULTS}

1... Experiments on newborn rats.

(a) Gross and microscopic findings concerning the experiments, in which the anti-serum was given perorally and the inhibitors were injected subcutaneously, are summarized in table 11 and 12 . In these experiments, the majority of the spleens were found during autopsy to be slightly or greatly enlarged.

(b) Gross and microscopic finding concerning the experiments, in which antirerum was injected subcutaneously and the inhibitors were injected subcutaneously, are summarized in table 13 and 14 . In these experiments the spleens were all greatly enlarged.

2. Gross and microscopic findings concerning the experiments on adult rats in -which both the anti-serum and inhibitors were injected subcutaneously, are 
Table 9

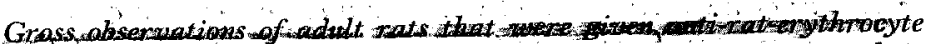
rabitit serum and the ratio of erythroblasts to leycocytes in their circulating blood and bone marrow smears

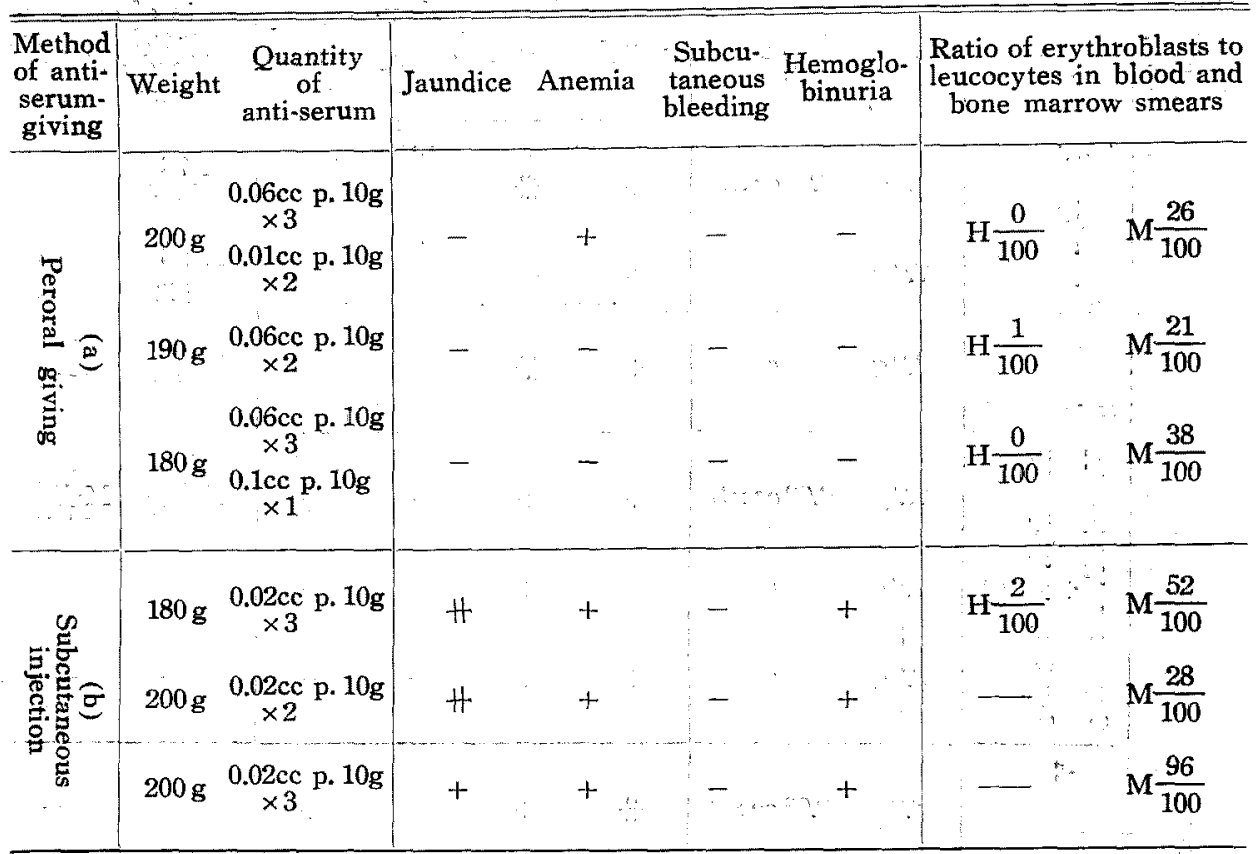

Table 10

Histologic findings in adult rats given anti-rat-erythrocyte rabbit serum perorally and subcutaneously

\begin{tabular}{|c|c|c|c|c|c|c|c|c|}
\hline \multirow[b]{2}{*}{$\begin{array}{l}\text { Method } \\
\text { of anti- } \\
\text { serum- } \\
\text { giving }\end{array}$} & \multicolumn{3}{|c|}{ Liver } & \multicolumn{2}{|c|}{ Kidney } & \multicolumn{2}{|c|}{ Spleen } & \multirow{2}{*}{$\begin{array}{c}\text { Bone } \\
\text { marrow } \\
\text { Increase } \\
\text { of erythro- } \\
\text { blasts }\end{array}$} \\
\hline & $\begin{array}{l}\text { Fatty } \\
\text { degenera- } \\
\text { tion }\end{array}$ & $\begin{array}{l}\text { Cloudy } \\
\text { swelling }\end{array}$ & $\begin{array}{l}\text { Increase } \\
\text { of } \\
\text { hemato- } \\
\text { poiesis }\end{array}$ & $\begin{array}{l}\text { Stagna- } \\
\text { tion of } \\
\text { glomeru- } \\
\text { lar tufts }\end{array}$ & $\begin{array}{c}\text { Cloudy } \\
\text { swelling of } \\
\text { convoluted } \\
\text { tublar- } \\
\text { epithelia }\end{array}$ & $\begin{array}{l}\text { Stagna- } \\
\text { tion }\end{array}$ & $\begin{array}{l}\text { Increase } \\
\text { of } \\
\text { erythro- } \\
\text { blasts }\end{array}$ & \\
\hline \multirow{3}{*}{ 量总 } & - & H & - & H & $H$ & + & H & + \\
\hline & - & + & - & + & + & + & + & - \\
\hline & - & H & - & + & + & $H$ & + & + \\
\hline \multirow{3}{*}{ 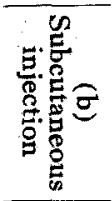 } & 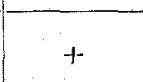 & - & - & $\downarrow$ & H & + & $\mathbb{H}$ & $H$ \\
\hline & - & H & - & - & $H$ & + & + & + \\
\hline & H & + & - & + & H & $H$ & $t$ & $H$ \\
\hline
\end{tabular}


Table 11

Gross observations of newborn rats that were given anti-rat-erythrocytes rabbit serum perorally and inhibitors subcutaneously and ratio of erythroblasts to leucocytes in their circulating blood and bone marrow smears

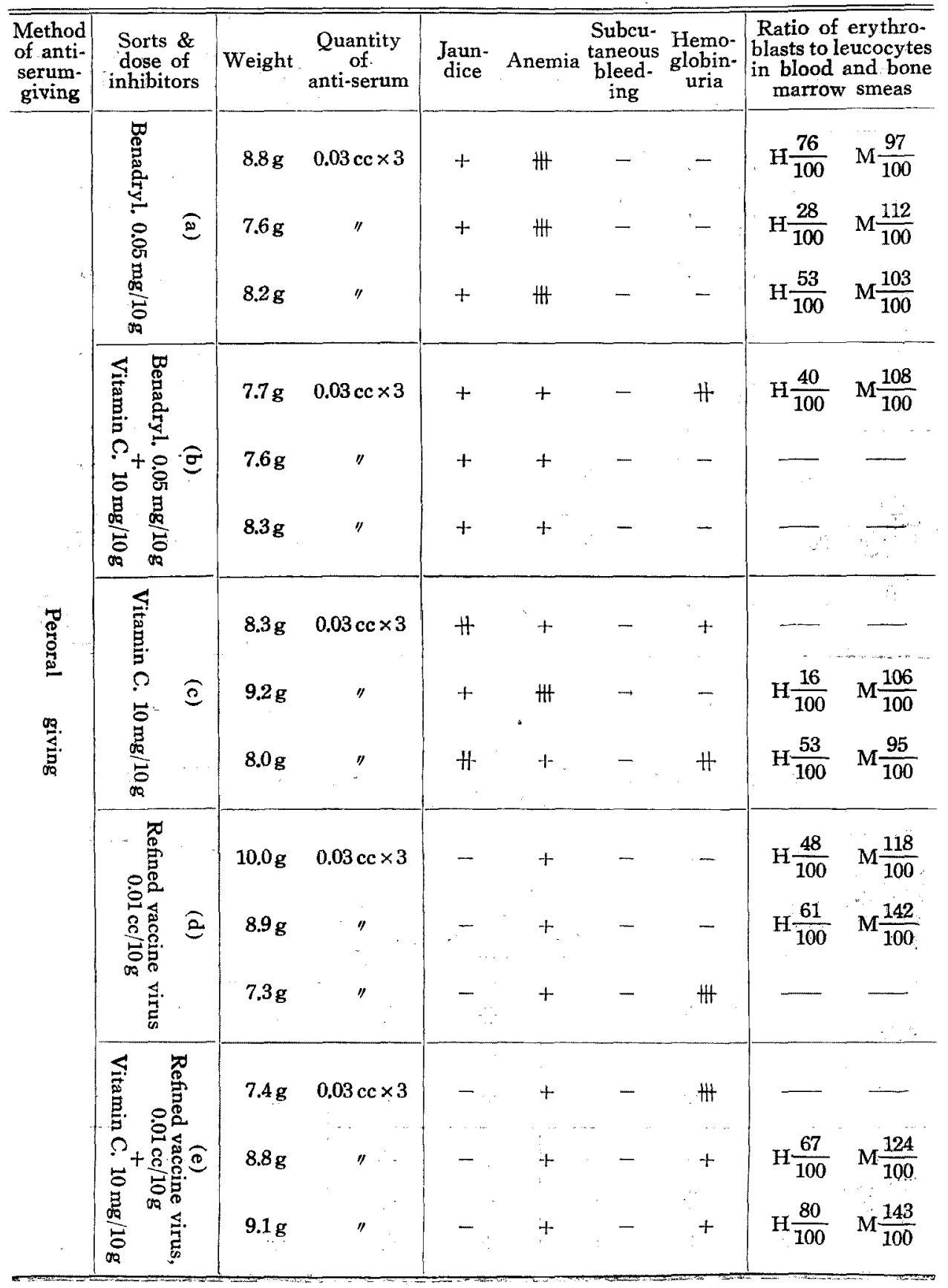


Table 11

Continued

\begin{tabular}{|c|c|c|c|c|c|c|c|c|c|}
\hline $\begin{array}{l}\text { Method } \\
\text { of anti- } \\
\text { serum- } \\
\text { giving }\end{array}$ & $\begin{array}{l}\text { Sorts \& } \\
\text { dose of } \\
\text { inhibitors }\end{array}$ & Weight & $\begin{array}{l}\text { Quantity } \\
\text { of } \\
\text { anti-serum }\end{array}$ & $\begin{array}{l}\text { Jaun- } \\
\text { dice }\end{array}$ & Anemia & $\begin{array}{l}\text { Subcu* } \\
\text { taneous } \\
\text { bleeding }\end{array}$ & $\begin{array}{l}\text { Hemo- } \\
\text { globin- } \\
\text { uria }\end{array}$ & $\begin{array}{c}\text { Ratio of } \\
\text { blasts to } 1 \\
\text { in blood } \\
\text { marrow }\end{array}$ & $\begin{array}{l}\text { erythro- } \\
\text { eucocytes } \\
\text { and bone } \\
\text { smears }\end{array}$ \\
\hline \multirow{4}{*}{$\stackrel{9}{\stackrel{9}{9}}$} & 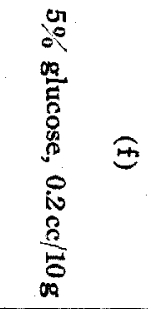 & $\begin{array}{l}12.4 \mathrm{~g} \\
14.2 \mathrm{~g} \\
13.8 \mathrm{~g}\end{array}$ & $\begin{array}{l}0.03 c c \times 3 \\
0.03 c c \times 1 \\
0.04 c c \times 2 \\
0.03 c c \times 1 \\
0.04 c c \times 2\end{array}$ & $\begin{array}{l}+ \\
+\end{array}$ & $\begin{array}{l}+ \\
+ \\
+\end{array}$ & - & $\begin{array}{l}- \\
- \\
-\end{array}$ & $\begin{array}{l}\mathrm{H} \frac{5}{100} \\
\mathrm{H} \frac{6}{100} \\
\mathrm{H} \frac{2}{100}\end{array}$ & $\begin{array}{l}M \frac{57}{100} \\
M \frac{64}{100} \\
M \frac{46}{100}\end{array}$ \\
\hline & 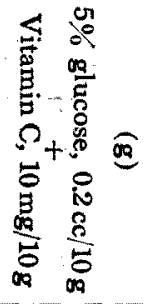 & $\begin{array}{l}13.0 \mathrm{~g} \\
14.5 \mathrm{~g} \\
18.8 \mathrm{~g}\end{array}$ & $\begin{array}{c}0.03 \mathrm{cc} \times 1 \\
0.04 \mathrm{cc} \times 2 \\
\therefore \\
\therefore\end{array}$ & $\begin{array}{l}+ \\
+\end{array}$ & $\begin{array}{l}+ \\
+ \\
+\end{array}$ & $\begin{array}{l}- \\
=\end{array}$ & $\begin{array}{l}- \\
\therefore- \\
\therefore=\end{array}$ & $\begin{array}{c}H \frac{13}{100} \\
H \frac{1}{100} \\
H \frac{3}{100}\end{array}$ & $\begin{array}{l}M \frac{63}{100} \\
M \frac{32}{100} \\
M \frac{65}{100}\end{array}$ \\
\hline & 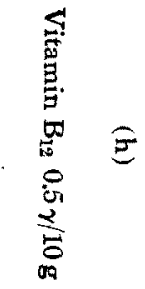 & $\begin{array}{l}11.8 \mathrm{~g} \\
13.5 \mathrm{~g} \\
15.3 \mathrm{~g}\end{array}$ & $\begin{array}{c}0.03 \mathrm{cc} \times 2 \\
0.04 \mathrm{cc} \times 1 \\
0.03 \mathrm{cc} \times 1 \\
0.04 \mathrm{cc} \times 2 \\
\\
\Rightarrow\end{array}$ & $\begin{array}{l}+ \\
+\end{array}$ & + & - & $\begin{array}{l}- \\
- \\
-\end{array}$ & $\begin{array}{l}H \frac{4}{100} \\
H \frac{1}{100} \\
H \frac{7}{100}\end{array}$ & $\begin{array}{l}M \frac{56}{100} \\
M \frac{67}{100} \\
M \frac{33}{100}\end{array}$ \\
\hline & 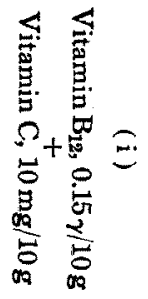 & $\begin{array}{l}13.5 \mathrm{~g} \\
10.1 \mathrm{~g} \\
13.7 \mathrm{~g}\end{array}$ & $\begin{array}{l}0.03 \mathrm{cc} \times 1 \\
0.04 \mathrm{cc} \times 2 \\
0.03 \mathrm{cc} \times 3 \\
0.03 \mathrm{cc} \times 1 \\
0.04 \mathrm{cc} \times 2\end{array}$ & + & + & - & - & $\begin{array}{l}H \frac{3}{100} \\
H \frac{18}{100} \\
H \frac{5}{100}\end{array}$ & $\begin{array}{l}M \frac{31}{100} \\
M \frac{60}{100} \\
M \frac{50}{100}\end{array}$ \\
\hline
\end{tabular}


Table 12

Histologic findings in newborn rats that were given anti-rat-erythrocyte rabbit serum perorally and inhibitors subcutaneously

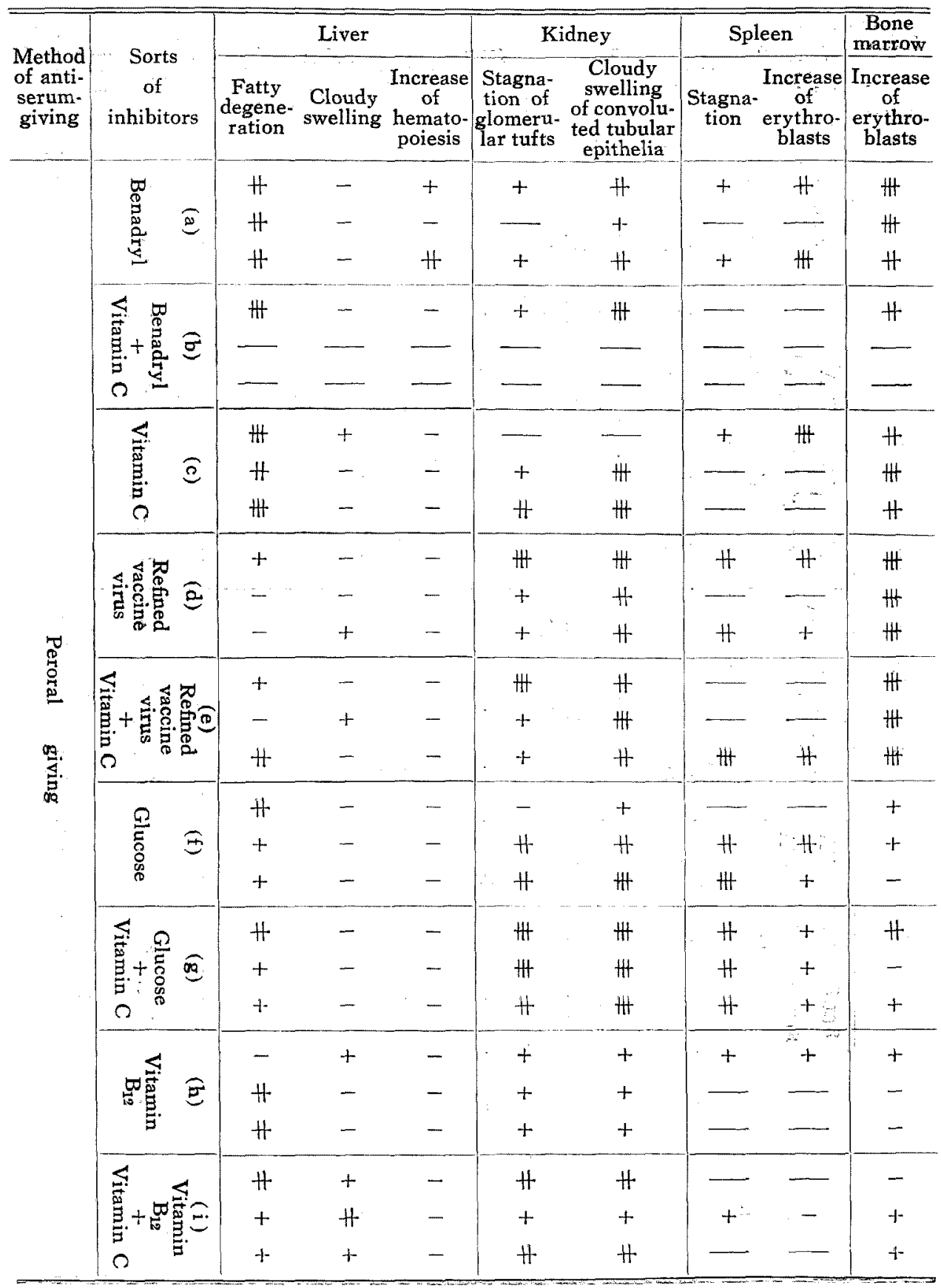


Table 13

Gross observations of newborn rats that were given anti-rat-zrythrocyte rabbit serum and inhibitors subcutaneously and ratio of erythroblasts to leucocytes in their circulating blood and bone marrow smears

\begin{tabular}{|c|c|c|c|c|c|c|c|c|c|}
\hline $\begin{array}{l}\text { Method } \\
\text { of-anti- } \\
\text { serum } \\
\text { giving } \\
\end{array}$ & $\begin{array}{c}\text { Sorts \& } \\
\text { dose of } \\
\text { inhibitors }\end{array}$ & Weight & $\begin{array}{c}\text { Quantity } \\
\text { of } \\
\text { anti-serum }\end{array}$ & $\begin{array}{l}\text { Jaun- } \\
\text { dice }\end{array}$ & Anemia & $\begin{array}{l}\text { Subcu- } \\
\text { taneous } \\
\text { bleeding }\end{array}$ & $\begin{array}{l}\text { Hemo- } \\
\text { globin- } \\
\text { uria }\end{array}$ & $\begin{array}{l}\text { Ratio of } \\
\text { blasts to } \\
\text { in blood } \\
\text { marrow }\end{array}$ & $\begin{array}{l}\text { erythro- } \\
\text { eucocytes } \\
\text { and bone } \\
\text { smears }\end{array}$ \\
\hline \multirow{7}{*}{ 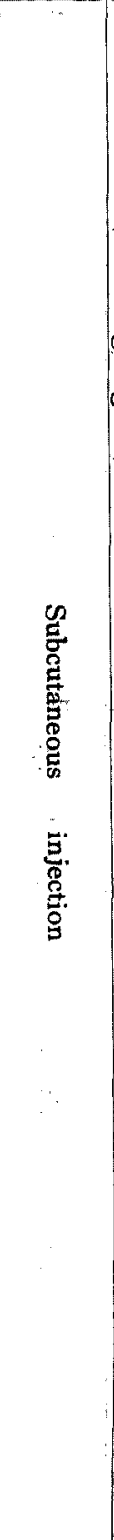 } & 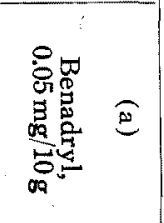 & $\begin{array}{l}10.5 \mathrm{~g} \\
10.6 \mathrm{~g} \\
12.0 \mathrm{~g}\end{array}$ & $\begin{array}{c}0.01 \mathrm{cc} \times 2 \\
" \\
"\end{array}$ & $\begin{array}{l}+ \\
+\end{array}$ & $\begin{array}{l}+ \\
+ \\
+\end{array}$ & - & - & $\begin{array}{l}\mathrm{H} \frac{11}{100} \\
\mathrm{H} \frac{7}{100} \\
\mathrm{H} \frac{21}{100}\end{array}$ & $\begin{array}{l}\mathrm{M} \frac{46}{100} \\
\mathrm{M}-\frac{96}{100} \\
\mathrm{M} \frac{82}{100}\end{array}$ \\
\hline & 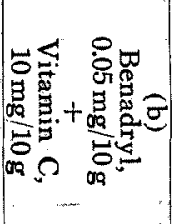 & $\begin{array}{r}10.3 \mathrm{~g} \\
9.7 \mathrm{~g} \\
11.0 \mathrm{~g}\end{array}$ & $\begin{array}{c}0.01 \mathrm{cc} \times 2 \\
" \prime\end{array}$ & H & $\begin{array}{l}+ \\
+ \\
+\end{array}$ & - & $\begin{array}{l}- \\
- \\
-\end{array}$ & $\begin{array}{l}\mathrm{H} \frac{17}{100} \\
\mathrm{H} \frac{16}{100} \\
\mathrm{H} \frac{13}{100}\end{array}$ & $\begin{array}{l}\mathrm{M} \frac{81}{100} \\
\mathrm{M} \frac{67}{100} \\
\mathrm{M} \frac{96}{100}\end{array}$ \\
\hline & 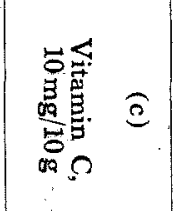 & $\begin{array}{r}10.2 \mathrm{~g} \\
9.3 \mathrm{~g} \\
10.0 \mathrm{~g}\end{array}$ & 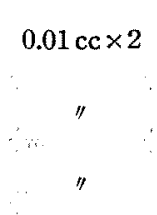 & $\begin{array}{l}H \\
H\end{array}$ & $\begin{array}{l}+ \\
+ \\
+\end{array}$ & $\begin{array}{l}- \\
- \\
-\end{array}$ & $\begin{array}{l}- \\
- \\
+\end{array}$ & $\begin{array}{l}\mathrm{H} \frac{8}{100} \\
\mathrm{H}+\frac{16}{100} \\
-\end{array}$ & $\begin{array}{l}M \frac{10}{100} \\
M \frac{55}{100} \\
\end{array}$ \\
\hline & 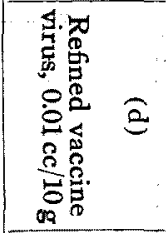 & $\begin{array}{l}12.6 \mathrm{~g} \\
11.0 \mathrm{~g} \\
12.7 \mathrm{~g}\end{array}$ & $\begin{array}{c}0.01 \mathrm{cc} \times 2 \\
"\end{array}$ & + & $\begin{array}{l}+ \\
+\end{array}$ & - & - & $\begin{array}{l}\mathrm{H} \frac{4}{100} \\
\mathrm{H} \frac{28}{100} \\
\mathrm{H} \frac{12}{100}\end{array}$ & $\begin{array}{l}M \frac{85}{100} \\
M \frac{37}{100} \\
M \frac{52}{100}\end{array}$ \\
\hline & 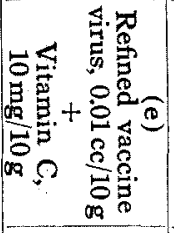 & $\begin{array}{r}10.5 \mathrm{~g} \\
9.7 \mathrm{~g} \\
10.7 \mathrm{~g}\end{array}$ & $\begin{array}{c}0.01 \mathrm{cc} \times 2 \\
"\end{array}$ & $\begin{array}{l}+ \\
+\end{array}$ & $\begin{array}{l}+ \\
+ \\
+\end{array}$ & - & $\begin{array}{l}- \\
+ \\
+\end{array}$ & $\begin{array}{l}\mathrm{H} \frac{9}{100} \\
\mathrm{H} \frac{11}{100}\end{array}$ & $\begin{array}{c}\mathrm{M} \frac{98}{100} \\
\mathrm{M} \frac{85}{100}\end{array}$ \\
\hline & 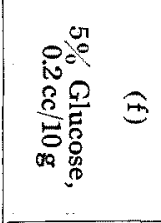 & $\begin{array}{r}10.6 \mathrm{~g} \\
\\
9.8 \mathrm{~g} \\
8.5 \mathrm{~g}\end{array}$ & $\begin{array}{c}0,01 \mathrm{cc} \times 2 \\
" \\
"\end{array}$ & H & + & - & $\begin{array}{l}+ \\
- \\
+\end{array}$ & $\begin{array}{l}\mathrm{H} \frac{12}{100} \\
\mathrm{H} \frac{6}{100} \\
\mathrm{H} \frac{39}{100}\end{array}$ & $\begin{array}{l}\mathrm{M} \frac{75}{100} \\
\mathrm{M} \cdot \frac{94}{100} \\
\mathrm{M} \cdot \frac{45}{100}\end{array}$ \\
\hline & 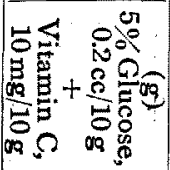 & $\begin{array}{l}7.3 \mathrm{~g} \\
8.9 \mathrm{~g}\end{array}$ & $0.01 c c \times 2$ & H & + & - & + & $\mathrm{H} \frac{24}{100}$ & $\mathrm{M} \frac{42}{100}$ \\
\hline
\end{tabular}


Table 14

Histologic findings in newborn rats that were given both anti-rat-erythrocyte rabbit serum and inhibitors subcutaneously

\begin{tabular}{|c|c|c|c|c|c|c|c|c|c|}
\hline \multirow[b]{2}{*}{$\begin{array}{l}\text { Method } \\
\text { of anti- } \\
\text { serum- } \\
\text { giving }\end{array}$} & \multirow[b]{2}{*}{$\begin{array}{c}\text { Sorts } \\
\text { of } \\
\text { inhibitors }\end{array}$} & \multicolumn{3}{|c|}{ Liver } & \multicolumn{2}{|c|}{ Kidney } & \multicolumn{2}{|c|}{ Spleen } & \multirow{2}{*}{\begin{tabular}{|c|} 
Bone \\
marrow \\
Inerease \\
of \\
erythro- \\
blasts
\end{tabular}} \\
\hline & & $\begin{array}{l}\text { Fatty } \\
\text { degene- } \\
\text { ration }\end{array}$ & $\begin{array}{l}\text { Cloudy } \\
\text { swelling }\end{array}$ & $\begin{array}{c}\text { Increase } \\
\text { of } \\
\text { hemato- } \\
\text { poiesis }\end{array}$ & $\begin{array}{l}\text { Stagna- } \\
\text { tion of } \\
\text { glomeru- } \\
\text { lar tufts }\end{array}$ & $\begin{array}{c}\text { Cloudy } \\
\text { swelling of } \\
\text { convoluted } \\
\text { tubular } \\
\text { epithelia }\end{array}$ & $\begin{array}{c}\text { Stagna- } \\
\text { tion }\end{array}$ & $\begin{array}{c}\text { Inerease } \\
\text { of } \\
\text { erythro- } \\
\text { blasts }\end{array}$ & \\
\hline \multirow{20}{*}{ 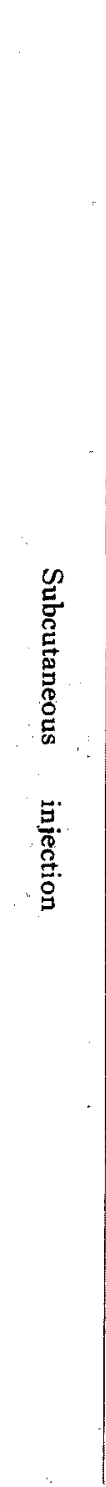 } & & + & - & + & H & H & - & - & + \\
\hline & 可 & + & - & - & - & + & - & $\longrightarrow$ & H \\
\hline & & + & - & + & + & + & + & + & $H$ \\
\hline & $\leq \varpi$ & $H$ & - & + & + & + & - & $\longrightarrow$ & H \\
\hline & $\frac{\mathrm{E}}{\mathrm{g}}+\mathrm{g}$ & $H$ & - & $t$ & + & $H$ & + & + & + \\
\hline & $2 \leftrightarrows$ & $H$ & - &,- & - & + & H & + & $H$ \\
\hline & & + & - & - & H & H & + & - & - \\
\hline & : & H & - & + & H & $H$ & H & + & - \\
\hline & & $H$ & - & + & + & H & + & $H$ & - \\
\hline & (4) & - & + & - & $H$ & H & $H$ & $H$ & $H$ \\
\hline & 量角 & $H$ & - & - & $H$ & H & + & H & - \\
\hline & $\frac{5}{6}$ & + & $H$ & - & $H$ & H & $H$ & + & - \\
\hline & $\leq: \stackrel{0}{9}$ & $H$ & - & - & $H$ & \# & $H$ & tt & - \\
\hline & 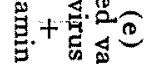 & + & - & - & H & H & $\mathbb{H}^{-}$ & - & $H$ \\
\hline & o & $H$ & - & - & - & - & - & $H$ & $H$ \\
\hline & & H & - & - & + & + & + & - & + \\
\hline & $\Phi$ & + & - & - & - & - & + & - & $H$ \\
\hline & & + & $H$ & + & + & + & + & + & - \\
\hline & $\lesssim \Omega$ & + & $H$ & + & + & + & H & $H$ & + \\
\hline & $\begin{array}{l}5 \\
5 \text {. } 80 \\
0 \\
0\end{array}$ & $H$ & $H$ & - & - & - & $H$ & - & - \\
\hline
\end{tabular}


Table 15

Gross observations of adult rats that were given both anti-rat-erythrocyte rabbit serum and inhibitors subcutaneously and the ratio of erythroblasts to leucocytes in their circulating blood and bone marrow smears

\begin{tabular}{|c|c|c|c|c|c|c|c|c|c|}
\hline $\begin{array}{l}\text { Method } \\
\text { of anti- } \\
\text { serum- } \\
\text { giving }\end{array}$ & $\begin{array}{l}\text { Sorts \& } \\
\text { dose of } \\
\text { inhibitors }\end{array}$ & Weight & $\begin{array}{c}\text { Quantity } \\
\text { of } \\
\text { anti-serum }\end{array}$ & $\begin{array}{l}\text { Jaun- } \\
\text { dice }\end{array}$ & Anemia & $\begin{array}{l}\text { Subecu: } \\
\text { taneous } \\
\text { bleeding }\end{array}$ & $\begin{array}{l}\text { Hemo- } \\
\text { globin- } \\
\text { uria }\end{array}$ & $\begin{array}{l}\text { Ratio of } \\
\text { blasts to I } \\
\text { in blood } \\
\text { marrow }\end{array}$ & $\begin{array}{l}\text { erythro- } \\
\text { eucocytes } \\
\text { \& bone } \\
\text { smears }\end{array}$ \\
\hline \multirow{2}{*}{ 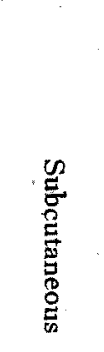 } & 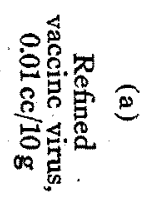 & $\begin{array}{l}190 \mathrm{~g} \\
200 \mathrm{~g}\end{array}$ & $\begin{array}{c}0.01 \mathrm{cc} \text { p. } 10 \mathrm{~g} \\
\times 2 \\
"\end{array}$ & $\begin{array}{l}+ \\
+\end{array}$ & + & - & + & $\begin{array}{l}H \frac{5}{100} \\
H \frac{4}{100}\end{array}$ & $\begin{array}{l}M \frac{24}{100} \\
M \frac{23}{100}\end{array}$ \\
\hline & 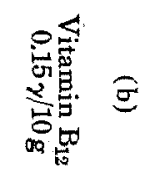 & $\begin{array}{l}170 \mathrm{~g} \\
190 \mathrm{~g}\end{array}$ & $\underset{\times 2}{0.01 c c \text { p. } 10 \mathrm{~g}}$ & $\begin{array}{l}\text { H } \\
H\end{array}$ & + & - & - & $\begin{array}{l}H \frac{16}{100} \\
H \frac{0}{100}\end{array}$ & $\begin{array}{l}M \frac{20}{100} \\
M \frac{20}{100}\end{array}$ \\
\hline$\therefore$ & 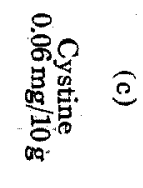 & $\begin{array}{l}190 \mathrm{~g} \\
180 \mathrm{~g}\end{array}$ & $\begin{array}{c}0.01 \mathrm{cc} \text { p. } 10 \mathrm{~g} \\
\times 2 \\
\text { " }\end{array}$ & + & + & - & + & $\begin{array}{l}H \frac{0}{100} \\
H \frac{6}{100}\end{array}$ & $\begin{array}{l}M \frac{17}{100} \\
M-\frac{36}{100}\end{array}$ \\
\hline
\end{tabular}

Table 16

Histologic findings in adult rats that were given both anti-rat-erythrocyte rabbit serum and inhibitors subcutaneously

\begin{tabular}{|c|c|c|c|c|c|c|c|c|c|}
\hline \multirow{2}{*}{$\begin{array}{l}\text { Method } \\
\text { of anti- } \\
\text { serum- } \\
\text { giving }\end{array}$} & \multirow[b]{2}{*}{$\begin{array}{c}\text { Sorts } \\
\text { of } \\
\text { inhibitors }\end{array}$} & \multicolumn{3}{|c|}{ Liver } & \multicolumn{2}{|c|}{ Kidney } & \multicolumn{2}{|c|}{ Spleen } & \multirow{2}{*}{$\begin{array}{c}\text { Bone } \\
\text { marrow } \\
\text { Increase } \\
\text { of } \\
\text { erythro- } \\
\text { blasts }\end{array}$} \\
\hline & & $\begin{array}{c}\text { Fatty } \\
\text { degene- } \\
\text { ration }\end{array}$ & $\begin{array}{l}\text { Cloudy } \\
\text { swelling }\end{array}$ & $\begin{array}{c}\text { Increase } \\
\text { of } \\
\text { hemato- } \\
\text { poiesis }\end{array}$ & $\begin{array}{l}\text { Stagna- } \\
\text { tion of } \\
\text { glomeru- } \\
\text { lar tufts }\end{array}$ & $\begin{array}{c}\text { Cloudy } \\
\text { swelling of } \\
\text { convoluted } \\
\text { tubular } \\
\text { epithelia }\end{array}$ & $\begin{array}{c}\text { Stagna- } \\
\text { tion }\end{array}$ & $\begin{array}{c}\text { Increase } \\
\text { of } \\
\text { ery thro- } \\
\text { blasts }\end{array}$ & \\
\hline \multirow{3}{*}{ 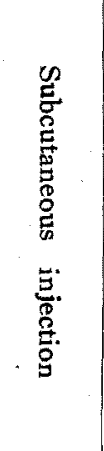 } & 起蛋 & - & + & - & $\begin{array}{l}H \\
+\end{array}$ & $\begin{array}{l}+1 \\
+\end{array}$ & $\begin{array}{l}\text { H } \\
H\end{array}$ & $\begin{array}{l}+ \\
+\end{array}$ & $\begin{array}{l}+ \\
+\end{array}$ \\
\hline & 可葛 & $\begin{array}{l}H \\
-\end{array}$ & H & $\begin{array}{l}- \\
-\end{array}$ & $\begin{array}{l}+ \\
+\end{array}$ & $\begin{array}{l}H \\
+\end{array}$ & $\begin{array}{l}\text { H } \\
\text { H }\end{array}$ & - & + \\
\hline & $\overbrace{0}^{\infty}$ & - & $\begin{array}{l}+ \\
+\end{array}$ & - & - & $\begin{array}{l}4 \\
+\end{array}$ & $\begin{array}{l}H \\
+\end{array}$ & $\begin{array}{l}- \\
+\end{array}$ & $\begin{array}{l}+ \\
+\end{array}$ \\
\hline
\end{tabular}


summarized in table 15 and 16. All the spleens were found to be greatly enlarged.

\section{DISGUSSION}

1. Pathologic changes very similar to the human hemolytic diseasc were produced by the hetero-immune sera between rat and rabbit that contained hemolysins. The appearance and the degree of these pathologic changes are proportional to the hemolysin titer of the anti-serum. With the fall in hemolysin titer these pathologic changes became difficult to be produced by giving the anti-serum enterally and not when the hemolysin titer fell to a certain degree. The fall in the hemolysin titer could not be supplemented by the increase in its volume.

2. By injecting the anti-erythrocyte serum which has the highest hemolysin titer among the anti-sera that were used in these experiments to adult rats, pathologic changes very similar to the so called hemolytic jaundice in human beings were developed, but the increase in the number of erythroblasts in their circulating blood was not recognized.

3. From these facts, it seems certified that the presumption which the $\mathrm{Rh}$ antibodies act in vivo as hemolysins is correct. At least one part of the similarity or identity between the pathogenesis of human hemolytic disease and of the so called hemolytic jaundice in human beings was demonstrated; the author believes that a clue for clarifying the pathogenesis of the so called human hemolytic jaundice was given.

4. From these facts, it can be said that congenital anemia is caused by the low immunization and icterus gravis by the high immunization of the mothers.

5. L. E. Young et $\mathrm{al}^{(6)}$. reported in their experiments on dogs that the newborn dogs do not absorb the antibodies after 1 to 2 days. The reason why the newborn dogs did not absorb the antibodies seems to be attributable to the sudden fall of hemolysin titers 1 to 2 days after delivery as can be found in the tables.

D. W. Bruner et al(7). reported in their experiments on pigs that baby pigs delivered from normal sow and allowed to nurse an immunized sow did not develop homolytic disease; that baby pigs delivered from immunized sow and raised with their-mother's milk developed hemolytic disease.

The reason why the normal baby pigs did not develop homolytic disease is attributable to the fact that when the baby pigs began to nurse, the sudden fall of antibody titer had already occurred. 
6. When the anti-erythrocyte serum was given to newborn rats perorally, refined vaccine virus and refined vaccine virus plus vitamine $G$ could inhibit the appearance of jaundice completely, but they could not when the antierythrocyte serum was given subcutaneously.

Since vitamine $\mathrm{C}$ only could not inhibit the appearance of jaundice, the inhibiting action of the refined vaccine virus plus vitamine $\mathrm{C}$ is attributable to the action of the refined vaccine virus only. In these cases degenerative changes of the liver were slighter compared with the others, but hemoglobinuria as more severe than in other cases. From these facts, it seems that the mechanism of inhibiting jaundice by using refined vaccine virus is attributable to the inhibition or impediment of the liver-disfunction, the rise in excretion power of the kindneys by exciting the reticuloendothelial system and impediment of homolysis to some degree by their adherence ${ }^{(8)}$ to hemolysin. The reason why jaundice could not be inhibited when anti-serum was given subcutaneausly is considered to be attributable to the rapidity of hemolysin absorption and its large quantity compared to peroral giving.

7. When the anti-erythrocyte serum was given to adult rats, jaundice could not be completely inhibited, but refined vaccine virus and cystine were able to inhibit it to some degree. In all these cases, the degenerative changes were slight. The inhibiting action of cystine seems to be attributable to the inhibition of liver damages, excitation of the reticuloendothelial system and its anti-dotal action.

8. In these experiments of newborn rats, extramedullary hematopoiesis was very slight except for the spleens. The reason for this seems to be attributable to the elapsed days after birth, being more in rats than in human.

E. L. Potter ${ }^{(9)}$ says that erythroblasts in circulating blood of a human hemolytic disease disappear rapidly in proportion to the lapse of hours after birth. The erythroblasts in circulating blood are not necessarily proportional to erythroblasts in the bone marrow. Tokoro(10) says that the erythroblast is not a sole indicator of the erythroblast in bone marrow.

\section{SUMMARY}

1. By peroral and parenteral giving of anti-erythrocyte serum and anti-serum serum and parenteral giving of anti-bone marrow serum, pathologic changes very similar to the human hemolytic disease were produced in newborn rats, but failed to do so in the peroral and parenteral giving of anti-liver serum, 
2. Pathologic changes very similar to the so-called hemolytic jaundice were demonstrated in adult rats by subcutaneous injection of anti-erythrocyte serum, but not by its peroral giving except for a slight increase of erythroblasts in the bone marrows of all cases and increase of erythroblasts in the bone marrows of all cases and slight anemia in one case.

3. When anti-erythrocyte serum was given to newborn rats perorally, refined vaccine virus and refined vaccine virus plus vitamine $C$ could inhibit the appearance of jaundice completely, but not when that anti-serum was given subcutaneously.

4. When anti-erythrocyte serum was given to adult rats, the appearance of jaundice could not be completely inhibited, while refined vaccine virus and cystine inhibited it to a certain degree.

5. The erythroblasts in circulating blood consisted of normoblasts of almost the same stage of development and their protolasma were orthochromatic or polychromatic except for some which were basophylic. The anisocytosis of the red cells was conspicuous and especially a remarkable increase in the number of microcytes was noticed.

\section{REFERENCES}

1. Bruner, D. W., Brown, R. G., Hull, F. E. and Kinkaid, A. S.: J. Am. Vet. M. A., 115, 869, 94-96, 1949.

2. Young L. E., Ervin, D. M., Christian, R. M. and Davis, R. W.: Science, 109, 630-631, 1949.

3. Young, L. E., Christian, R. M., Ervin, D. M., Davis, R. W., O'Brien, W. A., Swisher, S. N. and Yuile, C. L.: Blood, 6, 291-313, 1951.

4. Christian, R. M., Ervin, D. M. Swisher, S. N., O'Brien, W. A. and Young, L. E.: Science, 110, 443, 1949.

5. Bessis, M, and Frexia, P.: Rev. d'hemat. 2, 114-146, 1947. Cited in (3)

6. Same above (3).: 6, 308, 1951.

7. Same above (1):: 115, 869; 95, 1949.

8. Moriyama, H.: Virus no Hontai to Seimei no Kigen I; 42, 1950 (Japanese).

9. Potter, E. L.: Rh: Its Relation to Congenital Hemolytic disease and to Intragroup Transfusions Reactions, 16, 17, 1947.

10. Tokoro, Y.: Ketsuekigaku Togikai Hokoku II, 72 (Japanese). 